\title{
Pharmacocinétique de l'iode: revue des connaissances utiles en radioprotection accidentelle
}

\author{
B. GEOFFROY*, P. VERGER*, B. LE GUEN**
}

(Manuscrit resu le 6 juillet 1999, accepté le 19 octobre 1999)

RÉSUMÉ Lors d'une exposition à des rejets accidentels d'iodes radioactifs, la prise orale d'iode stable (iodure de potassium) permet de réduire ou d'éviter l'irradiation de la glande thyroïde. La posologie optimale est celle qui permet d'obtenir un blocage rapide et maximal du captage thyroïdien tout en évitant un dysfonctionnement thyroïdien. Cet article présente une revue des études sur la cinétique de l'iode dans l'organisme humain et l'efficacité du blocage thy roïdien par l'iode stable. Chez l'adulte euthyrö̈dien, la prise de $100 \mathrm{mg}$ d'iodure juste avant l'exposition permet d'éviter $95 \%$ ou plus de la dose à la thyroïde. Si l'exposition aux iodes radioactifs se prolonge après cette prise, le pourcentage de dose évitée à la thyroïde diminue de façon sensible. La répétition quotidienne de la prise d'iodure à une posologie plus faible (15 $\mathbf{m g})$ permet alors de maintenir un blocage du captage supérieur à $90 \%$. L'efficacité du blocage et la survenue d'un dysfonctionnement thyroïdien dépendent de facteurs externes ou individuels : apports alimentaires d'iode, état fonctionnel de la thyroïde et âge. I a posologie d'iode stable doit être adaptée en fonction de l'âge des individus au moment de l'exposition.

ABSTRACT Iodine kinetics and effectiveness of stable iodine prophylaxis after intake of radioiodine: a review.

Ingestion of stable iodine (potassium iodide) offers an efficient protection against the irradiation of the thyroid when an accidental exposure to radioiodine occurs. This prophylaxis aims at obtaining a rapid and maximum thyroid protection without antithyroid effects. This article reviews studies on iodine kinetics in the human and on stable iodine effectiveness to protect the thyroid. In adults with a normal thyroid function, ingestion of $100 \mathrm{mg}$ of iodide just before exposure to radioiodine allows a percentage of thyroid averted dose equal or greater than $95 \%$. If the exposure persists after iodide ingestion $(100 \mathrm{mg})$, the percentage of averted dose may decrease significantly. Repeated ingestion of daily amounts of 15 mg of stable iodine would then allow to maintain a $90 \%$ effectiveness. Iodide effectiveness and antithyroid effects also depend on external and individual factors such as iodine amounts in the diet, thyroid function and age. It is recommended to adapt the amount of ingested stable iodine according to age at the time of exposure.

\footnotetext{
* Institut de protection el de sâreté nucléaire, DPHD. SEGR/LEADS. BP 6, 92265 Fontenay-aux-Roses Cedex, France.

Mél. picre.verger (a) ipsn.fr

** Institul de protection el de sûreté nucléaire, OPHD, SDOS LEMDI, BP 6, 92265 Fontenay-aux-Roses Cedex, France.
} 


\section{Introduction}

La prise orale d'iode stable par les individus lors d'un rejet accidentel d'iode radioactif dans l'atmosphère a pour but de limiter voire d'empêcher la fixation d'iode radioactif par la glande thyroïde et de réduire ou éviter ainsi son irradiation. La glande thyroïde est en effet l'un des organes les plus radiosensibles de l'organisme (Ron et al., 1995). À la suite de l'accident de Tchernobyl, une augmentation sans précédent de la fréquence des cancers de la thyroïde a été observée chez l'enfant et l'adolescent en Biélorussie, Ukraine et Russie. L'exposition à l'iode 131 rejeté lors de l'accident conjuguée à l'absence de protection thyroïdienne ont joué un rôle vraisemblablement prédominant dans la survenue de cette augmentation, même si le rôle d'autres facteurs individuels et environnementaux ne peut être exclu (Bard et al., 1997).

Différents comités internationaux ont publié des recommandations pour l'administration d'iode stable en cas d'accident radiologique (ICRP 1991 ; IAEA 1994, 1996) et différents pays, dont la France, ont pris des dispositions pour organiser et préciser les modalités de mise en œuvre de cette mesure de protection (Piechowski, 1997). La planification de la prise d'iode stable lors d'un accident radiologique nécessite de connaître son efficacité et sa durée d'action en fonction des conditions d'administration et des caractéristiques individuelles ainsi que la nature et la fréquence des effets indésirables éventuels.

L'objectif de cet article est de présenter une synthèse des connaissances scientifiques et expérimentales disponibles sur la pharmacocinétique de l'iode dans l'organisme humain et sur le blocage du captage thyrö̈dien par l'iode stable et d'en dégager les paramètres utiles dans un but de gestion de santé publique. Les effets indésirables ne sont pas abordés.

\section{Rappel sur le métabolisme de l'iode}

L'iode est un élément indispensable à la synthèse des hormones thyroïdiennes, la triiodothyronine (T3) et la thyroxine (T4), qui sont synthétisées et sécrétées par la glande thyroïde. Il provient de l'alimentation et est absorbé au niveau du tube digestif (estomac, intestin grêle) sous forme réduite d'iodure $\mathrm{I}^{-}$(Tubiana et Schlumberger, 1997). Une fois dans le flux sanguin, il diffuse rapidement dans le secteur extracellulaire, constituant le pool d'iodure extracellulaire, et suit deux voies principales, en concurrence (Fig. 1):

- le captage par la glande thyroïde et la fabrication des hormones thyroïdiennes;

- la filtration par le glomérule rénal et l'élimination dans les urines.

Le captage de l'iodure par les cellules thyroidiennes est le résultat d'un transport actif régulé par une hormone sécrétée par l'hypophyse antérieure, la thyreo-stimuling- 


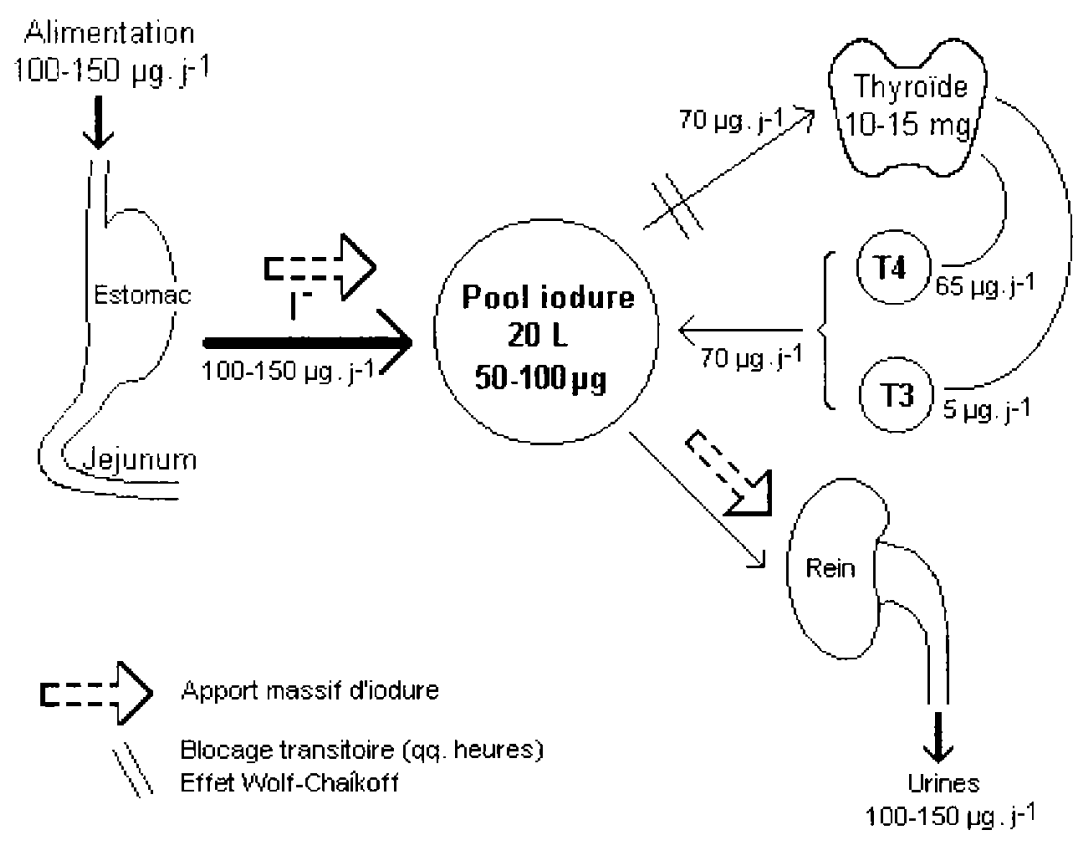

Fig. 1. - Modèle métabolique de l'iode chez l'homme (valeurs numériques pour un adulte).

Iodine human metabolic model (figures for an adult).

hormone (TSH) (Cavalieri, 1997). Le transport de l'iodure dans la thyroïde est l'étape limitante de l'accumulation de l'iode dans la thyroïde (Stanbury, 1990). Une fois capté, l'iodure est organifié c'est-à-dire oxydé puis combiné aux résidus thyrosine de la thyroglobuline, forme sous laquelle les hormones thyroïdiennes sont stockées dans la glande thyroïde (Cavalieri, 1997).

Les hormones thyroïdiennes sont libérées dans la circulation sanguine par protéolyse de la thyroglobuline, sous l'influence de la TSH. Lors de leur action au niveau des tissus cibles, l'iode est libéré et rejoint le secteur extracellulaire. Il peut être soit capté à nouveau par la glande thyroïde (recyclage) et servir à la synthèse hormonale soit être éliminé, pour l'essentiel, par le rein (Tubiana and Schlumberger, 1997). Le recyclage d'iode radioactif dans la thyroïde peut bien entendu entraîner une irradiation supplémentaire de celle-ci (Crocker, 1984).

En dehors de la glande thyroïde, l'iode se concentre aussi dans les glandes mammaires, les glandes salivaires, la muqueuse gastrique et le placenta (Cavalieri, 1997; Riggs, 1952). 


\section{Données cinétiques sur le métabolisme de l'iode}

Depuis l'utilisation d'isotopes radioactifs de l'iode comme traceurs dans les explorations fonctionnelles thyroïdiennes, de nombreuses études ont été publiées sur la cinétique d'incorporation de l'iode par l'organisme.

\subsection{Populations et méthodes utilisées}

Les études ont été effectuées sur des sujets volontaires sains recrutés lors d'une visite médicale systématique ou des sujets malades mais indemnes de toute pathologie connue pour interférer avec le métabolisme thyroïdien, ou enfin des sujets recrutés au sein d'institutions sanitaires ou scolaires. Dans tous les cas, les personnes traitées par des médicaments susceptibles de modifier le métabolisme thyroïdien, ou ayant eu une injection de produits de contraste radiologique ou ayant une pathologie thyroïdienne connue ont été exclues des études sauf, bien sûr, lorsque cette pathologie était étudiée.

On admet que les isotopes radioactifs de l'iode utilisés comme traceurs ont le même métabolisme que l'iode stable. L'iode 131 est un émetteur bêta, gamma ; il a été beaucoup utilisé car sa période radioactive de 8 jours permet de suivre ce traceur de façon prolongée. Mais cet isotope n'est dorénavant utilisé qu'à des fins thérapeutiques car il délivre une dose de rayonnement trop importante. L'iode 132, utilisé dans quelques études anciennes, a également été abandonné. Actuellement, le produit utilisé en médecine nucléaire est l'iode 125, un émetteur gamma : il irradie peu la glande car sa période physique n'est que de 13 heures.

Dans les études dont les résultats sont présentés ci-après, le traceur est le plus souvent administré par voie digestive, parfois par inhalation d'un aérosol constitué de gouttelettes d'une solution d'iode radioactif, exceptionnellement par voie intraveineuse. Les traceurs sont suivis depuis l'incorporation par l'organisme jusqu'à leur excrétion dans les urines. Avant l'administration d'un échantillon, son activité est mesurée. Les traceurs d'iode radioactif émettent des rayonnements bêta et gamma. La mesure de l'activité produite par le traceur incorporé dans la thyroïde, généralement des émissions gamma, est effectuée par un compteur externe placé en regard de la base du cou à une distance constante d'un sujet à l'autre pour une même expérience et avec une fenêtre de comptage de dimension fixe. Le calibrage du compteur est fait par la mesure d'échantillons d'activités connues placés à l'intérieur d'un corps thyroïdien factice. Un comptage externe est réalisé en regard de la cuisse pour permettre une estimation de l'activité extrathyroïdienne. Les mesures effectuées sont corrigées vis à vis de la décroissance radioactive de l'isotope administré.

\subsection{Absorption digestive}

Après administration orale, il n'y a pas de différence significative dans la cinétique d'incorporation de l'iodure ou de l'iodate chez l'homme. La réduction de ce dernier 
est quasi immédiate (Cronquist et al., 1971). Une étude chez le rat a montré que l'iodate et l'iodure de potassium avaient la même efficacité pour éviter le captage d'iode radioactif par la thyroïde (Pahuja et al., 1993).

L'absorption intestinale débute dès que l'iode arrive dans l'estomac. En effet, après l'administration orale d'iode 131, l'apparition du traceur radioactif a été observée en regard du cou dans un délai de 3 minutes environ, chez un sujet à jeun (Myant et Pochin, 1949).

L'absorption intestinale de l'iode est quasi complète chez l'adulte, l'adolescent et le jeune enfant. La CIPR recommande ainsi une valeur de 1 pour la fraction absorbée (ICRP, 1979; 1990). La présence d'aliments dans l'estomac retarde l'absorption d'environ 10-15 minutes (Keating et Albert, 1949; Kovari, 1994; Myant et Pochin, 1949). Chez pratiquement tous les sujets, l'absorption est complète 2 heures au maximum après l'ingestion (Keating et Albert, 1949; Tubiana et Schlumberger, 1997).

\subsection{Absorption pulmonaire}

L'incorporation d'iode administré par voie respiratoire dépend de la forme sous laquelle l'iode est inhalé et de la taille des particules de l'aérosol vecteur.

Les principales formes d'iode présentes dans un rejet radioactif accidentel provenant d'un réacteur de puissance sont les suivantes (Crocker, 1984):

- l'iode moléculaire (I2) présent sous forme gazeuse; après inhalation, la rétention dans l'arbre respiratoire est d'environ $100 \%$ et peu influencée par le rythme respiratoire; l'absorption dans le sang est rapide (demi-vie de 10 minutes) et complète (ICRP, 1995); Morgan et al. ont observé que la majorité de l'iode moléculaire se dépose dans l'oropharynx et très peu au niveau de la trachée et des bronches, l'iode n'atteignant pas les alvéoles pulmonaires (Morgan et al., 1968); l'iode est dégluti avec la salive et les sécrétions des voies aériennes supérieures et suit le métabolisme de l'iode ingéré ;

- l'iode dit organique, dont une forme courante est l'iodure de méthyle (ICH3), présent sous forme gazeuse; sa rétention pulmonaire est de $70 \%$ en moyenne (53 à $92 \%$ ) et elle augmente lorsque le rythme respiratoire diminue (Morgan et Morgan, 1967) ; l'absorption sanguine est très rapide (demi-vie de 5 secondes) (ICRP, 1995);

- l'iode particulaire (aérosol) : le diamètre de ses particules est variable, cellesci pouvant s'agréger entre elles; la rétention pulmonaire est la plus élevée pour les particules ayant un diamètre de moins d'un micron ; en fait, l'incorporation de l'iode est complète quelle que soit la taille des particules car ce qui ne pénètre pas dans l'arbre respiratoire est dégluti et absorbé au niveau digestif ; l'absorption dans le sang est également rapide (demi-vie de 10 minutes chez le singe) (ICRP, 1995). 
Les proportions respectives de ces différentes formes dans un rejet accidentel dépendent du scénario d'accident et de l'importance relative des voies de sortie de l'enceinte du réacteur (fuites directes, fuites collectées, rejet à travers le filtre à sable...). Par exemple, le filtre à sable est très efficace vis-à-vis des aérosols mais ne retient pas l'iode organique.

\subsection{Captage thyroüdien et clairance thyroüdienne}

Après ingestion unique d'iode radioactif, le captage thyroïdien est d'abord rapide puis atteint un plateau. Chez les sujets ayant une fonction thyroïdienne normale, ce plateau est atteint en 24 à 48 heures environ et correspond à une fraction de $10 \%$ à $40 \%$ de l'iode total ingéré (Keating et Albert, 1949; Kovari, 1994). La demi-vie, c'est-à-dire le temps au bout duquel la moitié de cette fraction est captée par la thyroïde, varie dans ces mêmes études entre 3 et 6,5 heures (Kovari, 1994). Les variations interindividuelles sont importantes (Berson et al., 1952).

Le captage dépend de la clairance thyroïdienne et de la concentration plasmatique d'iode selon la formule suivante (Berson et al., 1952):

captage thyroïdien $(\mu \mathrm{g} / \mathrm{min})=$ clairance thyroïdienne $(\mathrm{ml} / \mathrm{min}) \times$ concentration plasmatique $(\mu \mathrm{g} / \mathrm{ml})$.

La clairance thyroïdienne est la quantité de plasma épurée de l'iodure par la thyroïde par unité de temps. Elle dépend du volume de la glande et de son activité. Elle est adaptative : elle dépend de la concentration de l'iodure plasmatique et des apports alimentaires d'iode. Chez le sujet normal, elle a été évaluée à 16$18 \mathrm{ml} / \mathrm{min}$ (Berson et al., 1952; Myant et Pochin, 1949).

\subsection{Elimination rénale}

Le rein est la voie principale d'élimination de l'iode dont l'essentiel est excrété sous forme d'iodure (Riggs, 1952). Après ingestion d'un traceur, l'apparition de radioactivité dans les urines est quasi immédiate (Keating et Albert, 1949). L'excrétion rénale de l'iode est rapide dans les premières heures et atteint un plateau au bout de 24 à 48 heures (Berson et al., 1952; Keating et Albert, 1949; Myant et Pochin, 1949).

L'iode qui n'est pas capté par la thyroïde est retrouvé en intégralité dans les urines des 24 à 48 heures suivant l'incorporation, soit environ la moitié à deux tiers environ de l'iode ingéré. À l'état normal, l'iode présent dans les urines des 24 heures est un excellent reflet des apports totaux quotidiens en iode alimentaire (Tubiana et Schlumberger, 1997). 
La clairance rénale, quantité de plasma épurée de l'iodure par le rein par unité de temps, n'est pas ou très peu influencée par les apports en iode : elle est non adaptative et non saturable (Berson et al., 1952; Stanbury, 1990). Elle varie entre 30 et $50 \mathrm{ml}$ de plasma par minute.

\subsection{Autres voies d'élimination}

Une faible partie de l'iode organique est éliminée dans les fêces sous forme de thyroxine conjuguée dans le foie et sécrétée dans la bile. En état de fonctionnement normal de la glande thyroïde, elle représente au maximum $20 \%$ de la production de thyroxine quotidienne.

Dans l'espèce humaine, l'iode est également concentré dans le lait maternel selon un gradient de 20 à 30 ou plus (Stanbury, 1990). Il y est présent presque exclusivement sous forme inorganique. La plus grande partie est excrétée dans le lait dans les 48 heures suivant l'ingestion (Weaver et al., 1960). Lorsque la production de lait par la mère est importante, elle représente une voie majeure d'excrétion de l'iode par l'organisme. Le transfert d'iode dans le lait maternel peut être bloqué par une surcharge iodée (Stanbury, 1990).

\subsection{Facteurs de variation du captage thyroïdien}

Il existe une grande variabilité dans la cinétique du métabolisme de l'iode selon les individus. Les études expérimentales ont permis de dégager les principaux facteurs expliquant ces variations.

\subsubsection{Apports alimentaires d'iode}

Les besoins quotidiens en iode dans des conditions normales sont estimés entre 100 et $200 \mathrm{mg}$ chez l'homme adulte, selon les auteurs (Delange et al., 1997; Wolff, 1969) et sont de $30 \mathrm{mg}$ chez le nouveau-né (Delange, 1990a). Les apports alimentaires en iode ont une influence directe sur le captage thyroïdien de l'iode (Fig. 2). En effet, celui-ci augmente lorsque les apports d'iode sont plus faibles (Crocker, 1984; Kaul et al., 1980; Parker et Beierwaltes, 1962; Saxena et al., 1962; Zvonova, 1989) pour maintenir le captage d'une quantité totale d'iode suffisante pour assurer la fonction thyroïdienne.

La figure 2 montre que le captage thyroïdien d'iode passe de $40 \%$ pour des apports quotidiens de $100 \mathrm{mg} / \mathrm{j}$ à près de $60 \%$ lorsque ces apports ne sont plus que de $50 \mathrm{mg} / \mathrm{j}$, situation fréquemment rencontrée en France (Mornex, 1987; Valeix et Hercberg, 1992). Lors d'une exposition à des iodes radioactifs, la dose à la thyroïde augmente en proportion. 


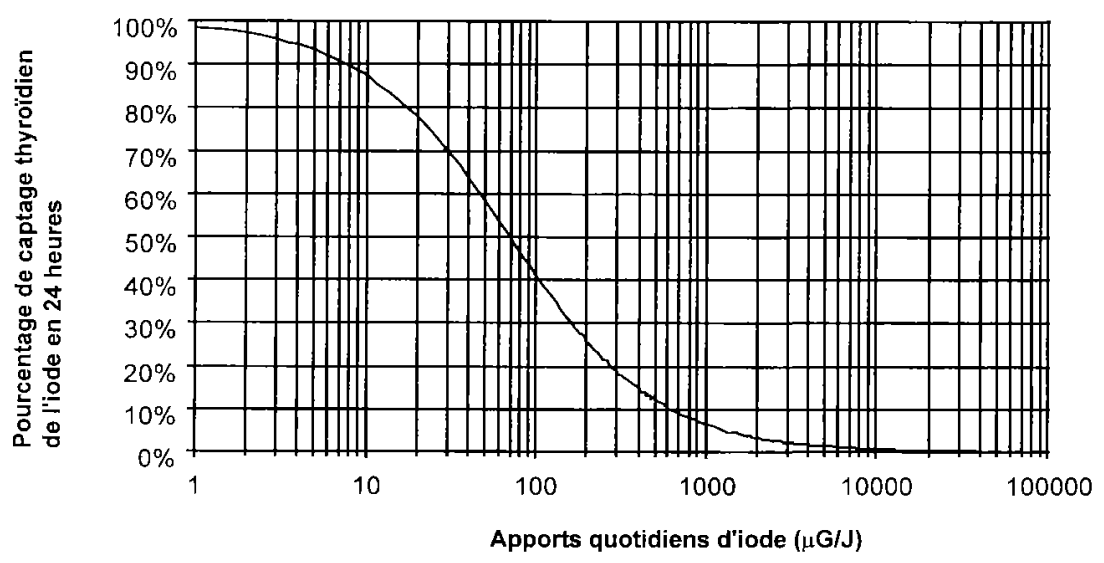

Fig. 2. - Taux de captage de l'iodure plasmatique par la thyroide en fonction des apports quotidiens d'iode ( $\mu \mathrm{g})$ (Malarbet et al., 1998).

Thyroid plasmatic iodide uptake fraction as a function of daily iodine intake $(\mu g)$ (Malarhet et al., 1998).

\subsection{2. Âge}

Durant la grossesse, l'iode traverse la barrière placentaire (Stanbury, 1990). L'exposition maternelle à l'iode radioactif entraîne donc une exposition du foetus dont les conséquences sont variables en fonction du terme.

Le captage d'iode par la thyrö̈de fotale commence vers $10-12$ semaines (Evans, et al., 1967 ; Hodges et al.; 1955, Roti et al., 1983) et reste faible jusqu'à 22 semaines. Après 22 semaines, elle augmente rapidement jusqu'au terme (Evans et al., 1967). Lors d'une exposition maternelle à l'iode radioactif durant cette période, sa concentration dans la thyroïde foetale sera plus élevée que dans la thyroïde maternelle car le pool d'iode fœtal est beaucoup plus faible que celui de la mère (van Middlesworth, 1963).

Concernant les enfants, plusieurs études ne montrent pas de différence de captage par rapport aux adultes (Cuddihy, 1966 ; Oliner et al., 1957). Chez l'adolescent, en revanche, se produit une accélération des échanges d'iode: la clairance et le captage thyroïdiens ainsi que la clairance rénale sont augmentés et la demi-vie de l'iode au sein de la thyroïde est plus courte que pour l'adulte (Malvaux, 1985).

La plupart des études réalisées chez l'adulte montrent une baisse progressive du captage thyroïdien avec l'âge, indépendamment du sexe (Gaffney et al., 1962; Rosenberg, 1957; Schober et Hunt, 1976 ; Tubiana et al., 1958) qui correspond à une altération progressive des structures fonctionnelles de la glande (Mochizuki 
et al., 1963). De même, la clairance rénale de l'iode diminue régulièrement avec l'âge (Gaffney et al., 1962).

\subsubsection{Sexe}

Une étude chez l'adulte euthyrö̈dien montre une légère différence, statistiquement significative, de captage thyrö̈dien d'iode 131 entre l'homme et la femme (respectivement $23,5 \%$ et $25,8 \%$ à 24 heures) (Quimby et al., 1950). Mais ce résultat n'est pas constamment retrouvé (Tubiana et al., 1958). Une étude réalisée chez des sujets âgés de 2 mois à 18 ans ne montre pas de différence en fonction du sexe (Oliner et al., 1957).

\subsubsection{Femme enceinte}

L'augmentation physiologique de la filtration glomérulaire et la baisse de la réabsorption tubulaire des iodures sous l'effet des oestrogènes chez la femme enceinte ont pour conséquence une fuite d'iode par les urines ce qui entraîne une baisse du pool iodé avec une carence relative en iode si les apports ne sont pas augmentés (Valeix et Hercberg, 1992). On observe alors une augmentation compensatrice de la clairance thyroïdienne d'autant plus marquée que les apports initiaux en iode alimentaire sont faibles. La réduction de la quantité d'iode disponible pour la mère s'aggrave au cours de la seconde moitié de la grossesse car une fraction de l'iode ingéré est captée par l'unité foeto-placentaire.

\subsubsection{Anomalies thyroüdiennes}

En cas d'hyperthyroïdie, la clairance thyroïdienne et le captage maximal peuvent être fortement modifiés ; par exemple, ils augmentent dans la maladie de Basedow (environ $500 \mathrm{ml} / \mathrm{min}$ dans l'étude de Myant and Pochin (1949). À l'inverse, la clairance rénale ne semble pas influencée.

En cas de goitre euthyroïdien d'origine carentielle, les taux plasmatiques et urinaires d'iode sont abaissés et la clairance thyroïdienne augmentée (Koutras et al., 1960). La quantité totale d'iode capté reste semblable à celle de sujets normaux. Tout se passe comme s'il existait un mécanisme compensateur permettant de préserver le captage d'une quantité normale d'iode en augmentant l'extraction par le tissu thyroïdien.

Chez les adultes, les pathologies thyroïdiennes ne sont pas rares. Une étude effectuée au Royaume Uni permet d'estimer, chez la femme et l'homme respectivement, la prévalence des hyperthyroïdies à $3,9 \%$ et $0,2 \%$ et celle des goitres à $12,3 \%$ et $0,9 \%$ (Wang et Crapo, 1997). 


\section{Blocage thyroïdien par l'iode stable}

\subsection{Mécanismes d'action de l'iode stable}

Aucun agent ne permet, par un mécanisme direct, d'accélérer l'élimination de la radioactivité concentrée au sein de la glande thyroïde'. Elle persiste plusieurs semaines et décroît au fur et à mesure de la sécrétion hormonale (ICRP, 1979).

Il est donc fondamental en cas d'exposition à l'iode radioactif, de prévenir la contamination de la glande. Divers agents antithyroïdiens empêchent le captage d'iode radioactif par la glande (Crocker, 1984). Parmi eux, l'iode stable est efficace et moins susceptible d'induire des effets secondaires (Tubiana et Schlumberger, 1997).

Le captage et l'organification de l'iode par la thyroïde croissent proportionnellement à la concentration plasmatique, le rapport iode thyroïdien/iode plasmatique restant constant (Stanbury, 1990). Une surcharge iodée brutale entraîne :

- un phénomène de saturation et de dilution isotopique ;

- une inhibition aiguë de l'organification de l'iode et de la synthèse hormonale;

- une inhibition de la sécrétion hormonale thyroïdienne.

\subsection{Saturation et dilution isotopique}

Le phénomène de saturation et de dilution isotopique est le mécanisme principal sur lequel repose l'action protectrice de l'iode stable : celui-ci entre en compétition avec l'iode radioactif vis à vis du système de transport actif de l'iode dans la thyroïde. Cependant l'iode radioactif peut encore pénétrer dans la glande par diffusion (NCRP, 1977). La saturation par l'iode stable évite aussi le recyclage de l'iode radioactif organique au niveau de la thyroïde et diminue ainsi sa demi-vie effective (Lengemann et Thompson, 1963).

\subsection{Inhibition de l'organification de l'iode et de la synthèse hormonale}

Au-delà d'une concentration plasmatique d'iode de $15-28 \mathrm{mg} / \mathrm{dl}$, on observe une diminution rapide de l'organification de l'iode qui se traduit par une diminution de la synthèse hormonale (Delange, 1990b; Stanbury, 1990; Wolff, 1969): il s'agit de l'effet Wolff-Chaikoff, du nom des auteurs qui l'ont observé chez le rat en 1948.

\footnotetext{
${ }^{1}$ En-dehors d'injections répétées de TSH qui accélèrent la sécrétion hormonale.
} 
Ce processus, non encore parfaitement élucidé, est indépendant de la TSH. Il serait déterminé par la baisse du rapport iode thyroïdien/iode libre, notamment par augmentation importante de la concentration plasmatique en iode (Delange, 1990b; Stanbury, 1990).

Pour une thyroide adulte normale, l'effet Wolff-Chaikoff est transitoire. Il apparaît quelques heures après une charge iodée et dans les 24 à 48 heures, on observe une reprise de l'organification de l'iode par la thyroïde même si la surcharge iodée persiste (échappement) (Wolff, 1969).

L'inhibition de l'organification pourrait jouer un rôle protecteur complémentaire en empêchant le stockage d'iode radioactif sous forme organifiée dans la thyroïde (Orgiazzi, 1996).

Mais l'effet bloquant de l'organification induit par l'iode peut être plus important et durable et entraîner alors des effets secondaires thyroïdiens. Cela peut, en particulier, se produire lorsque le pool iodé thyroïdien est abaissé ou lorsque le niveau préalable du captage de l'iode par la thyroïde est augmenté : situations de carence iodée, thyroïdites, hyperthyroïdie, thyroïdectomie partielle, jeune âge (Wolff, 1969).

Le foetus est particulièrement sensible à l'effet Wolff-Chaikoff : le pool d'iode thyroïdien est faible et le captage élevé. Le phénomène d'échappement à l'effet Wolff-Chaikoff n'apparaît que tardivement, à partir de trente-six semaines de gestation environ (Wolff, 1969). Une surcharge iodée aiguë de l'ordre de 6 à 10 fois l'apport iodé normal chez le nouveau-né peut entraîner une hypothyrö̈die nécessitant un traitement hormonal substitutif (Castaing et al., 1979; Delange, 1991). Des cas d'hypothyroïdie néonatale sévère et parfois de goitres ont été décrits après application cutanée d'antiseptiques iodés (par exemple Povidone-iodine) au moment de l'accouchement, ou après des examens à l'aide de produits de contraste (amniofoetographie) durant la grossesse (Castaing et al., 1979; Clemens et Neumann, 1989; Delange, 1990b; Noteboom et al., 1997b; van Middlesworth, 1963).

Cependant, après la distribution d'iode stable en Pologne à la suite de l'accident de Tchernobyl, des tests thyroïdiens effectués à la naissance sur un échantillon de 3214 nouveau-nés dont la mère avait bénéficié d'une prophylaxie $(70 \mathrm{mg}$ d'iodure de potassium) n'ont détecté une hypothyroïdie biologique que chez 12 d'entre eux $(0,37 \%)$. Ces anomalies avaient disparu entre 16 et 20 jours (Nauman et Wolff, 1993). 
B. GEOFFROY et al.

\subsection{Inhibition de la sécrétion hormonale thyroüdienne}

L'inhibition de la sécrétion hormonale thyroïdienne, par inhibition de l'hydrolyse de la thyroglobuline, s'installe rapidement à des niveaux de concentration plasmatique inférieurs à ceux qui entraînent la diminution de la synthèse hormonale (Wolff, 1969). Cet effet est transitoire et ne dure pas plus de 7 jours (Delange, 1990b). Il est utilisé dans le traitement des hyperthyroïdies aiguës.

L'inhibition de la sécrétion hormonale thyroïdienne pourrait favoriser l'irradiation de la thyroïde si l'iode stable était ingéré lorsque de l'iode radioactif s'est déjà concentré dans la thyroïde (Crocker, 1984). Cependant, l'essentiel de la dose de rayonnements étant délivré dans les premiers jours suivant l'exposition, il est peu probable que cet effet aurait un impact significatif sur la dose de rayonnement à la thyroïde (Stanbury, 1990).

\subsection{Principales études sur l'action préventive de l'iode stable}

Une recherche bibliographique a été effectuée pour identifier les études sur le captage thyroïdien après administration d'iode stable. Les principales sont présentées ci-après :

- le captage thyroïdien a été mesuré 2 heures et 24 heures après l'administration orale d'iode $131\left(3,7 \times 10^{5} \mathrm{~Bq}\right)$ chez des adultes des deux sexes indemnes de toute pathologie susceptible d'interférer avec le métabolisme thyroïdien (Koutras et Livadas, 1966) ; la population de l'étude comportait 10 témoins et 60 sujets répartis en dix groupes recevant différentes posologies d'iode stable $(5,10,20,40,80$ mg d'iodure de potassium) soit en même temps que la prise du traceur soit 2 ou 12 heures après;

- le captage thyrö̈dien à 24 heures a été mesuré sur 62 volontaires sains, 37 hommes et 25 femmes, âgés entre 21 et 72 ans après administration d'une dose orale de $55 \mathrm{~Bq}$ d'iode 131 (Blum et Eisenbud, 1967); les sujets étaient leurs propres témoins; deux de ces sujets étaient hypothyroïdiens et ont été exclus de l'étude; l'iode stable a été administré à 24 hommes et 17 femmes, 1 heure avant, en même temps que, ou 1 à 6 heures après l'administration du traceur; les posologies étaient de 5, 25, 50, 100, 200, ou $1000 \mathrm{mg}$ d'iodure de potassium; chez ces sujets, une dose supplémentaire de traceur de 55 à $275 \mathrm{~Bq}$ a été administrée 24 à 72 heures après la prise d'iode stable pour évaluer la durée du blocage du captage thyroïdien;

- dans une étude sur 22 volontaires euthyrö̈diens ( 12 hommes et 10 femmes) âgés entre 23 et 50 ans, le captage d'iode $123\left(3,7 \times 10^{5} \mathrm{~Bq}\right)$ a été étudié à l'état basal puis après administration simultanée d'iodure de potassium (posologie de 10 , 30,50 et $100 \mathrm{mg}$ ) (Sternthal et al., 1980); la prise d'iode stable a été répétée pour la plupart des sujets pendant les 11 jours suivants; le captage a été mesuré à 3 heures, 6 heures puis 24 heures après la première prise d'iode stable puis au huitième et au douzième jour (Sternthal et al., 1980); 
- une étude a été réalisée en 1991 sur 29 sujets euthyroïdiens âgés entre 32 et 74 ans dont 13 femmes et 16 hommes (Pupi et al., 1991); six heures après une injection de $18,5 \times 10^{5} \mathrm{~Bq}$ d'iode 131 , de l'iodure de potassium était administré à la moitié du groupe à la posologie de $100 \mathrm{mg}$ et un placebo était administré à l'autre moitié ;

- la seule étude portant sur des enfants a été effectuée en 1961 dans le Massachusetts (États-Unis) sur 63 enfants souffrant d'un retard mental, sans anomalie thyroïdienne (Saxena et al., 1962) ; les enfants étaient répartis selon 3 groupes d'âge ( $1-3$ ans, 4-6 ans et 9-1 I ans) et recevaient de l'iode stable chaque jour pendant plusieurs semaines (posologies de 0,$1 ; 1 ; 3$ et $6 \mathrm{mg}$ par jour).

D'autres études ont été effectuées sur des effectifs plus faibles (Adams et Bonnell, 1962 ; Cronquist et al., 1971 ; Cuddihy, 1966; Johnson, 1963 ; Pochin et Barnaby, 1962 ; Ramsden et al., 1967).

Enfin, l'efficacité du blocage thyroïdien par l'iode stable a été calculée par simulation au moyen de modèles pharmacocinétiques (Adams et Bonnell, 1962; Wootton et Hammond, 1978; Zanconino et Becker, 1993).

\subsection{Résultats}

\subsubsection{Efficacité en fonction de la posologie d'iode stable}

Le blocage du captage thyroïdien varie en fonction de la posologie d'iodure de potassium administrée (Fig. 3). Un pourcentage de dose évitée ${ }^{2}$ de plus de $90 \%$ est obtenu chez l'adulte pour une posologie de l'ordre de $20 \mathrm{mg}$ lorsque l'administration a lieu au même moment que l'exposition aux iodes radioactifs (Blum et Eisenbud, 1967; Koutras et Livadas, 1966; Ramsden et al., 1967; Wootton et Hammond, 1978; Zanconino et Becker, 1993). Une concentration plasmatique d'iode de $10 \mathrm{mg} / \mathrm{dl}$ induit un blocage efficace du captage thyroïdien (Blum et Eisenbud, 1967).

Il convient néanmoins de souligner que la posologie minimale entraînant un blocage du captage thyroïdien dépend des caractéristiques individuelles: elle est plus importante chez les sujets ayant un captage thyroïdien élevé que chez les autres (Ramsden et al., 1967).

Des variations inter-individuelles existent pour les posologies d'iodure de 100 et $200 \mathrm{mg}$ administrées simultanément au traceur (Fig. 4) mais le pourcentage de dose évitée à la thyroïde, 24 heures après l'ingestion du traceur, est supérieur à

\footnotetext{
${ }^{2}$ Le pourcentage de dose évitée est à peu près égal au pourcentage de réduction de captage thyroïdien. La fraction évitée est calculée de la façon suivante :

(captage thyroïdien sans blocage - captage thyroïdien avec blocage) $\times 100 /$ captage thyroïdien sans blocage.
} 
B. GEOFFROY et al.

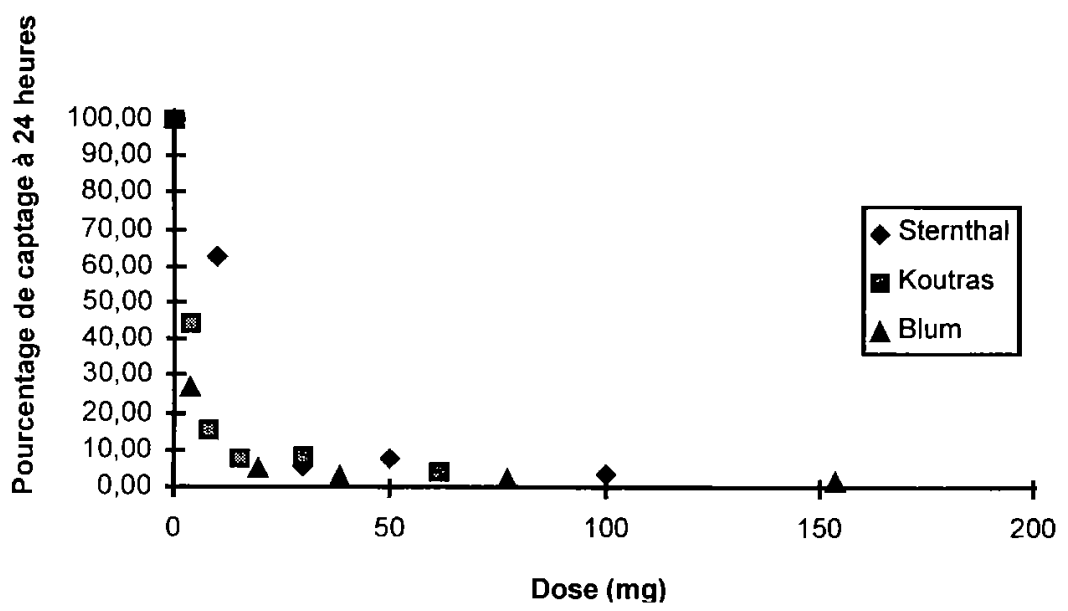

Fig. 3. - Pourcentage de captage thyroïdien selon la posologie d'iode stable administrée.

Thyroid iodide uptake according to the amount of ingested stable iodine.

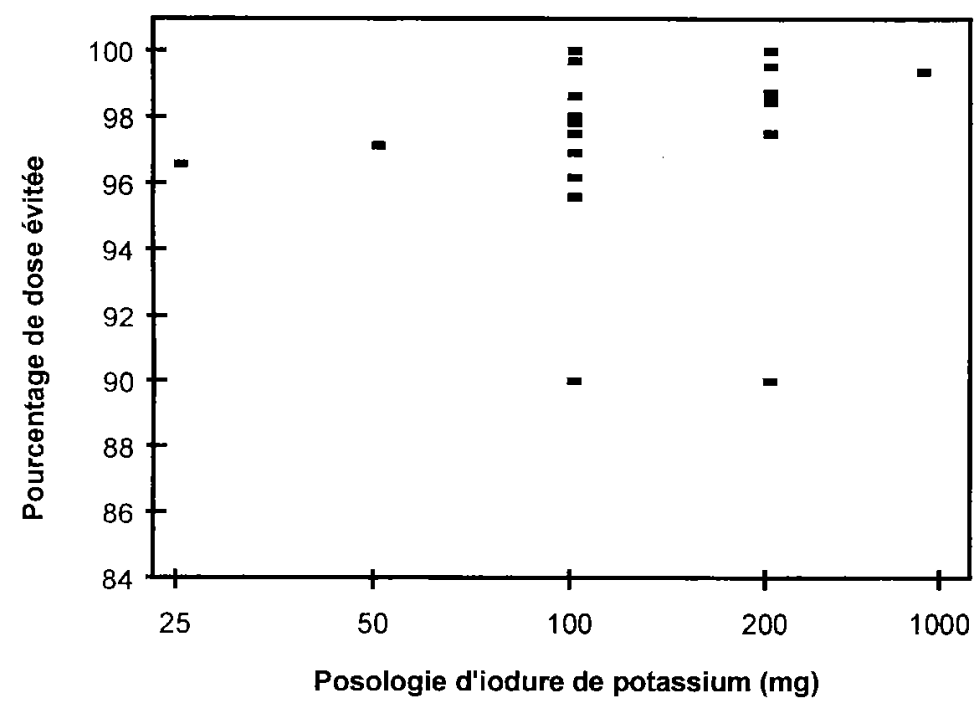

Fig. 4. - Pourcentage de dose évitée à la thyroüde en fonction de la posologie d'iodure de potassium (prise simultanée de l'administration du traceur) d'après Blum et Eisenbud (1967).

Percentage of thyroid averted dose according to the amount of ingested iodine (simultaneous ingestion of the radioactive tracer), Blum and Eisenbud (1967). 


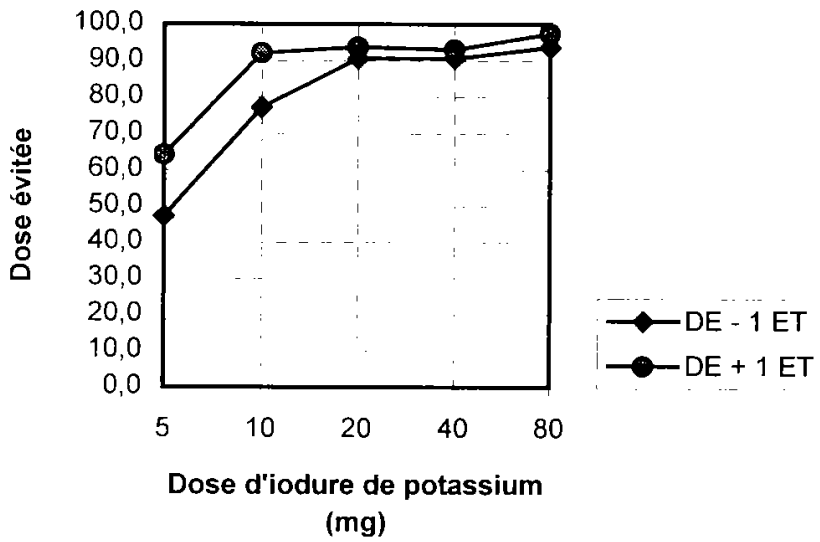

$D E-E T:$ dose moyenne évitée moins I déviation standard ; $D E+E T:$ dose movenne évitée plus $I$ déviation standard

Fig. 5. - Pourcentage de dose évitée à la thyroüde en fonction de la posologie d'iodure de potassium (prise simultanée de l'administration du traceur) d'après Koutras et Livadas (1966).

Percentage of thyroid averted dose according to the amount of ingested iodine (simultaneous ingestion of the radioactive tracer), Koutras and Livadas (1966).

95\% pour la plupart des sujets (Blum et Eisenbud, 1967). L'augmentation des posologies d'iode stable au-dessus de 100-200 mg ne semble plus améliorer la dose évitée (Koutras et Livadas, 1966) (Fig. 5).

Des posologies d'iodure de 3 à $4 \mathrm{mg}$ chez l'enfant ne permettent d'atteindre un blocage suffisant du captage thyroïdien qu'au bout de quelques jours à quelques semaines (Saxena et al., 1962).

\subsubsection{Efficacité en fonction du moment de l'administration de l'iode stable}

L'efficacité de l'iodure de potassium est maximale si l'administration d'iode stable a lieu en même temps ou un peu avant l'exposition à l'iode radioactif. L'efficacité décroît ensuite quand l'iode stable est administré quelques heures après l'ingestion du traceur radioactif.

Blum et al. observent que $100 \mathrm{mg}$ d'iodure de potassium permettent d'éviter $98 \%$ de la dose à la thyroïde lorsque la prise a lieu au même instant que le traceur, $91 \%$ si elle a lieu $1 \mathrm{~h}$ plus tard, $84 \% 2 \mathrm{~h}$ plus tard et $60 \% 3$ heures après le 
traceur (Blum et Eisenbud, 1967). L'augmentation de la posologie ne permet pas de corriger la diminution d'efficacité due au retard de la prise.

Une simulation, basée sur le modèle pharmacocinétique de Berman et al. (1968) fournit une estimation du pourcentage de dose évitée à la thyroïde en fonction du moment de la prise d'iode stable par rapport à l'exposition chez des sujets ayant des apports normaux en iode et chez des sujets ayant un déficit d' apport en iode (Fig. 6) (Zanconino et Becker, 1993). Une autre étude, basée sur le modèle compartimental de Ramsden et al. (1967) fournit des résultats très similaires et montre que le moment optimum de la prise d'iode stable est une heure avant l'exposition à l'iode radioactif (Wootton et Hammond, 1978) (Tab. 1).
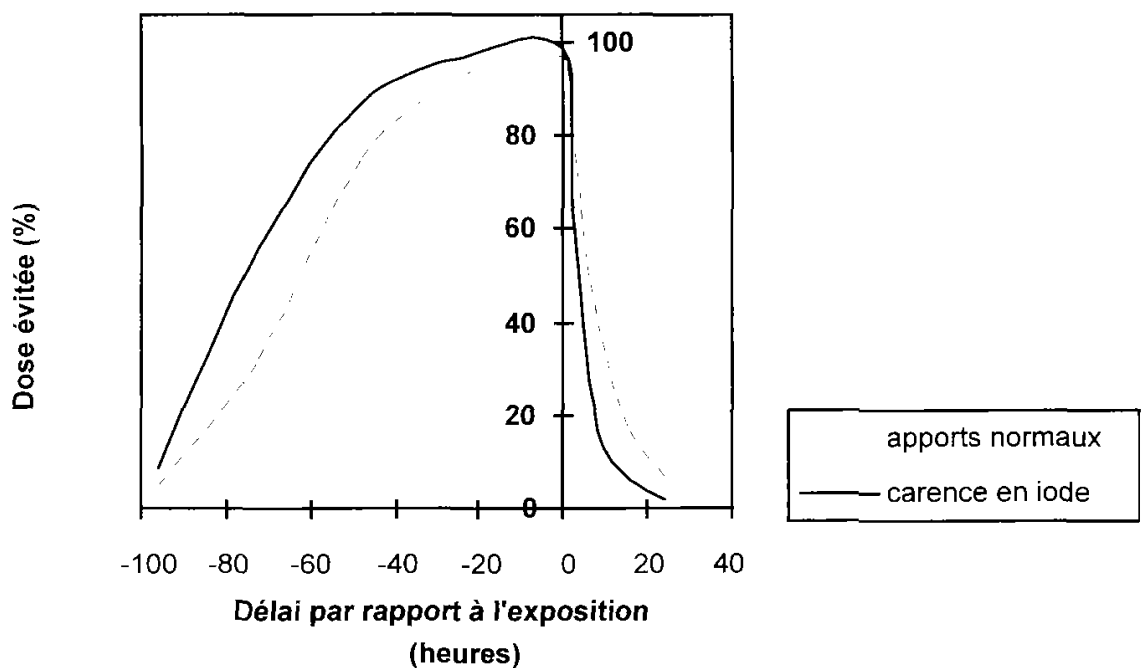

Fig. 6. - Pourcentage de dose évitée en fonction du délai de la prise d'iode stable par rapport à l'exposition. Simulation, prise d'iode stable unique, exposition unique (Zanconino et Becker, 1993).

Percentage of thyroid averted dose according to the delay between stable iodine ingestion and exposure. Simulation, unique exposure and stable iodine ingestion (Zanconino and Becker, 1993).

\section{TABLEAU 1}

Pourcentage de dose évitée en fonction du moment de la prise d'iode stable* : simulation (Wootton et Hammond, 1978).

Percentage of averted dose according to the time of stable iodine* administration: simulation (Wootton and Hammond, 1978).

\begin{tabular}{|c|c|c|c|c|c|c|c|}
\hline 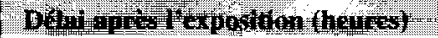 & 0 & 4 & 5 & 6 & 10 & 15 & 20 \\
\hline 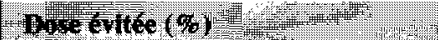 & 98 & 64 & 57 & $5 \mathrm{l}$ & 34 & 20 & 12 \\
\hline
\end{tabular}

* $128 \mathrm{mg}$ diodure 


\subsubsection{Durée de la protection}

La durée du blocage du captage thyrö̈dien est d'autant plus courte que la posologie initiale est faible (Zanconino et Becker, 1993).

Blum et al. ont étudié la durée du blocage du captage thyroïdien 48 et 72 heures après la prise d'iode stable en réadministrant aux sujets une dose d'iode radioactif (Blum et Eisenbud, 1967). Une posologie de $25 \mathrm{mg}$ n'assurait plus de blocage après 48 heures ; des posologies de $50 \mathrm{mg}$ et $100 \mathrm{mg}$ d'iodure de potassium permettaient d'éviter $66 \%$ et $78 \%$, respectivement, de la dose à la thyroïde. À 72 heures, une posologie de $100 \mathrm{mg}$ d'iode stable ne permettait d'éviter que $25 \%$ environ de la dose à la thyroïde (Tab. II, Fig. 7).

La durée de protection a été étudiée sur une période de 8 jours sur un échantillon de 5 femmes et 5 hommes indemnes de pathologie thyroïdienne ou rénale et hospitalisés pour ulcère gastrique ou infarctus myocardique (Johnson, 1963). Ces patients n'avaient pas reçu de médication contenant de l'iode. Le captage thyrödien a été mesuré chaque jour 3 heures après prise orale d'iode 132 . Une première détermination a été effectuée après administration de $200 \mathrm{mg}$ d'iodure de potassium.

\section{TABLEAU II}

Durée du blocage du captage thyrö̈dien en fonction de la posologie d'iode stable.

Duration of the blockade of iodine uptake by the thyroid gland according to stable iodine amount.

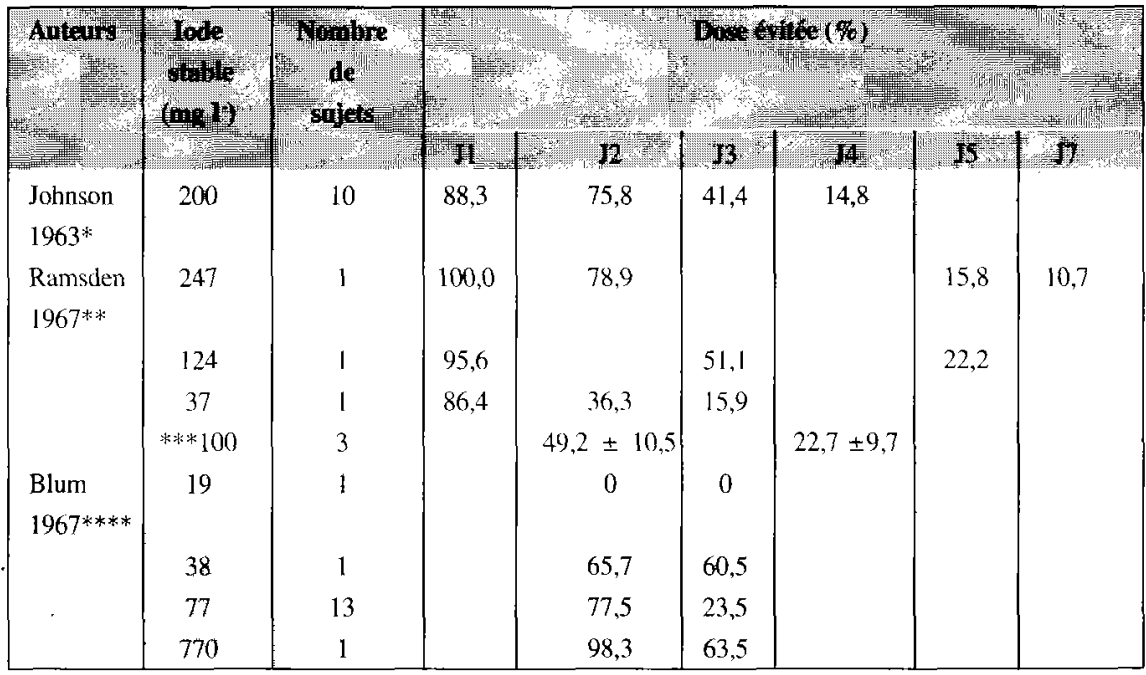

* captage à 3 heures. *: captage à 30 heures. **** administration de l'iode stable 1 h 30,3 ho0 et 5 h30 après le traceur pour chaque individu respectivement. 水永* captage à 24 heures, évaluation graphique de la dose évitée. 

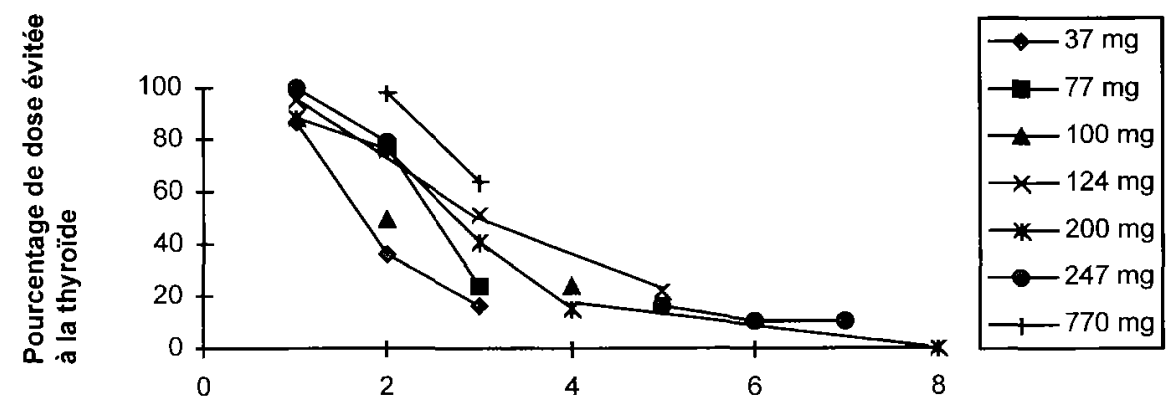

Temps après la prise d'iode stable (j)

Fig. 7. - Durée du blocage du captage thyroüdien en fonction de la posologie d'iodure (prise unique).

Thyroid blokade duration according to the amount of ingested stable iodide.

Les résultats de cette étude montrent que la dose évitée est supérieure à $75 \%$ dans les deux premiers jours suivant la prise d'iode stable, passe en dessous de $50 \%$ au troisième jour et à $15 \%$ au quatrième jour (Tab. II, Fig. 7). Le captage thyroïdien retrouve sa valeur de base au bout de huit jours chez la majorité des sujets.

Ramsden et al. ont mesuré le captage thyroïdien chez trois sujets ayant reçu un mélange d'iode $132\left(444 \times 10^{4} \mathrm{~Bq}\right)$ et d'iode $131\left(14,8 \times 10^{4} \mathrm{~Bq}\right)$ à différents moments après l'administration orale de différentes posologies d'iodure de potassium (Ramsden et al., 1967). Plusieurs déterminations du captage thyroïdien ont été faites dans le temps; les résultats sont présentés au tableau 2 pour le captage à 30 heures.

La modélisation des données obtenues montre également que le blocage du captage thyroïdien est effectif au bout d'une demi-heure environ après une prise d'iode stable de $100 \mathrm{mg}$ d'iodure de potassium. Ce délai n'est pas raccourci pour une posologie supérieure, mais il peut en revanche augmenter à plus de 2 heures 30 pour une posologie inférieure à $25 \mathrm{mg}$ d'iode stable (Ramsden et al., 1967).

Il existe enfin des données sur le prolongement de la durée de l'efficacité par l'administration quotidienne d'iode stable. L'étude de Sternthal et al. montre qu'une dose évitée de plus de $90 \%$ peut être maintenue par la prise répétée plusieurs jours de suite de $15 \mathrm{mg}$ d'iodure de potassium (Sternthal, 1980).

Cuddihy et al. ont administré à 4 sujets euthyroïdiens une dose orale de $18,5 \mathrm{~Bq}$ d'iode 131 pendant 14 jours (Cuddihy, 1966). Deux d'entre eux, âgés de 8 et 9 ans, recevaient également, chaque jour de la même période, $1,8 \mathrm{mg}$ d'iodure; les deux autres, âgés de 22 et 23 ans, recevaient de la même façon 4,2 $\mathrm{mg}$ 
d'iodure. Le pourcentage de dose évitée était de $33 \%$ et $48 \%$ pour les deux premiers sujets respectivement et de $69 \%$ et $62 \%$ pour les deux derniers.

\subsubsection{Efficacité en fonction du mode d'administration}

L'efficacité du blocage du captage thyroïdien par application cutanée de teinture d'iode a été montrée chez l'animal et chez l'homme. Dans une étude effectuée sur 24 hommes âgés entre 24 et 51 ans, le captage thyroïdien après la prise orale d'iodure de potassium (130 mg) a été comparé à celui mesuré chez des sujets chez lesquels de la teinture d'iode avait été appliquée sur l'avant-bras ( $4 \mathrm{ml}$ à $2 \%$ soit $80 \mathrm{mg}$ ) ou sur l'abdomen ( $8 \mathrm{ml}$ à $2 \%$ soit $160 \mathrm{mg}$ ) (Miller et al., 1989). Le pourcentage moyen de dose évitée était respectivement de $96,9 \%, 35,8 \%$ et $81,7 \%$.

\subsubsection{Efficacité en fonction de l'âge}

Les seules données dont on dispose sur l'efficacité du blocage du captage thyroïdien chez le fæutus en fonction de la posologie d'iode stable administrée à la mère proviennent d'études animales.

Une étude expérimentale a été conduite chez le chimpanzé pour évaluer l'efficacité du blocage thyroïdien par l'iode stable chez le fotus entre 19 et 21 semaines de grossesse (Noteboom et al., 1997a). L'iodure était administré par voie orale 1 heure avant le traceur. Les posologies d'iodure étaient de $0,5 \mathrm{mg} / \mathrm{kg}, 1,5 \mathrm{mg} / \mathrm{kg}$ et $5,0 \mathrm{mg} / \mathrm{kg}$. Ces posologies correspondent, pour un sujet de $50 \mathrm{~kg}$, à $25 \mathrm{mg}, 75 \mathrm{mg}$ et $250 \mathrm{mg}$ d'iodure. À titre de comparaison, la posologie recommandée chez la femme enceinte est de $100 \mathrm{mg}$ d'iodure.

En tout, cinq animaux ont reçu ces posologies. Le captage thyroïdien basal a aussi été mesuré chez 13 animaux témoins. En l'absence d'iode stable, le captage thyroïdien du traceur à $24 \mathrm{~h}$ chez le fœtus du chimpanzé était similaire à celui du foetus chez l'homme. Les résultats montrent que les trois posologies permettaient d'obtenir un pourcentage de dose évitée supérieur à $90 \%$. Mais, 20 heures après l'administration d'iode stable, le pourcentage de dose évitée n'était supérieur à $90 \%$ que pour les deux posologies les plus élevées alors que pour la posologie la plus faible, il était de $40 \%$.

\section{Conclusion}

\section{Principes de l'administration d'iode stable à visée préventive en cas de rejet accidentel d'iode radioactif}

L'administration d'iode stable permet d'éviter l'irradiation de la thyroïde en cas d'exposition à des rejets accidentels d'iodes radioactifs. Une surcharge iodée permet en 
effet de dépasser un certain seuil de concentration plasmatique et d'inhiber le transport de l'iode dans la thyroïde. La posologie optimale recherchée dans cette optique est celle qui permet d'obtenir un blocage rapide et maximal du captage thyroïdien (effet préventif) tout en évitant ou minimisant les risques d'effets secondaires, notamment les effets «antithyroïdiens» (effet Wolff-Chaikoff). Cet effet est susceptible d'entrâ̂ner une hypothyroïdie transitoire chez les sujets sensibles, notamment le nouveau-né.

\section{Efficacité de la protection thyrö̈dienne par l'iode stable}

Dans la plupart des études sur la protection thyroïdienne par l'iode stable passées en revue dans cet article, les méthodes de sélection des sujets ne sont pas ou peu décrites et le nombre de sujets est limité. Si l'on peut s'interroger sur leur représentativité, les résultats semblent cependant assez convergents.

L'efficacité du blocage du captage thyroïdien dépend des modalités d'administration de l'iode stable. Un blocage quasi immédiat ( 30 minutes après la prise) et efficace (pourcentage de dose évitée à la thyroïde égal ou supérieur à $90 \%$ ) peut être obtenu chez l'adulte euthyrö̈dien à partir d'une posologie d'iode stable relativement faible (20 mg) lorsque l'administration a lieu au même moment que l'exposition aux iodes radioactifs. Une posologie plus faible permet de bloquer la thyroide mais dans un délai plus long (plusieurs heures). Une posologie plus élevée permet d'atteindre une efficacité de $95 \%$ et de diminuer les variations inter-individuelles.

Le blocage du captage thyroïdien est transitoire et sa durée dépend de la posologie. Ce point est important pour les situations d'exposition prolongée ou retardée par rapport à la prise d'iode stable: lors d'un accident sur un réacteur de puissance, le moment précis du rejet et sa durée ne peuvent être prédits avec certitude. Pour une posologie voisine de 100 à $200 \mathrm{mg}$ d'iodure, le pourcentage de dose évitée à la thyroïde si une exposition a lieu 48 heures après la prise d'iode stable est compris entre 50 et $75 \%$.

En cas d'exposition prolongée, la répétition de la prise d'iode stable permet de maintenir un blocage efficace du captage thyroïdien au-delà de $24-48$ heures. Une posologie plus faible ( $15 \mathrm{mg}$ par jour) que lors de la première prise semble alors suffire pour assurer une protection satisfaisante chez l'adulte euthyroïdien (Sternthal et al., 1980).

L'efficacité du blocage dépend de la date de la prise par rapport à celle de l'exposition :

- l'efficacité est maximale si la prise d'iode stable a lieu 1 à quelques heures avant l'exposition à l'iode radioactif ;

- elle diminue si l'iode stable est pris quelques heures après l'exposition : la dose évitée est de $50 \%$ seulement si la prise d'iode stable a lieu 6 heures après l'exposition. 


\section{Facteurs individuels de variation de l'efficacité de l'iode stable}

L'efficacité du blocage et les risques d'effets secondaires thyroïdiens dépendent aussi de facteurs individuels (âge, contenu de la thyroïde en iode, état fonctionnel de la thyrö̈de) et de facteurs externes (apports alimentaires d'iode). Chez l'enfant, la glande thyroïde est plus avide pour l'iode radioactif que celle d'un adulte. Elle est également plus sensible à l'irradiation par l'iode radioactif, en termes de risques de cancer, que chez l'adulte (Bard et al., 1997, Shore, 1992). Enfin, clle est plus sensible à l'effet Wolff-Chaikoff que la glande thyroïde de l'adulte. La marge entre la posologie d'iodure permettant de protéger la thyroïde et celle risquant de provoquer des effets secondaires thyroïdiens est ainsi plus étroite que chez l'adulte.

Par ailleurs, les données existantes en France suggèrent que les apports alimentaires d'iode sont inférieurs aux recommandations de l'OMS pour quelques pour-cent à quelques dizaines de pour-cent des sujets dans tous les groupes d'âges (Valeix et Hercberg, 1992). En cas d'exposition à un rejet accidentel d'iode radioactif ceci peut, pour les individus concernés, augmenter les doses de rayonnement à la glande thyroïde et favoriser les effets secondaires thyroïdiens.

Les études présentées dans cet article ne permettent pas toujours d'obtenir une estimation quantitative des variations de l'efficacité du blocage du captage thyroïdien en fonction de ces facteurs individuels et externes. En particulier, les données chez le foetus, le nourrisson et l'enfant sont très limitées, pour des raisons éthiques évidentes. L'efficacité du blocage en fonction des apports alimentaires en iode n'est pas non plus bien connue.

La posologic d'iode stable doit être adaptée selon l'âge. Compte tenu de la sensibilité particulière des nouveau-nés à l'effet antithyroïdien de l'iode, il est recommandé de ne pas dépasser une posologie de $12,5 \mathrm{mg}$ et de procéder à un suivi hormonal rigoureux si la posologie administrée atteignait ou dépussait $25 \mathrm{mg}$ (WHO/CEC, 1990).

Remerciements: Les auteurs remercient le Pr. André Aurengo, chef du Service central de médecine nucléaire du groupe hospitalier Pitié-Salpêtrière et le Dr Jean Piechowski, Institut de protection et de sûreté nucléaire, Département de la protection de la santé de l'homme et de dosimétrie, pour leurs discussions et leur relecture attentive de l'article.

\section{RÉFÉRENCES}

Adams C.A., Bonnell J.A. (1962) Administration of stable iodine als a means of reducing thyroid irradiation resulting from inhalation of radioactive iodine. Health Phisics 7, 127-49.

Bard D., Verger P., Hubert P. (1997) Chernobyl, 10 ycars alter: Health consequences, Epidemiologic Rer. 19, 187-204.

Berman M., Hoff E., Barandes M., Becker D.B., Sonenberg M., Benua R., Koutras D.A (1968). Iodine kinetics in man-a model, J. Clin. Endocrinol. Metab. 28, 1-14.

Berson S.A., Yalow R.S., Sorrentino J., Roswit B (1952). The determination of thyroidal and renal plasma II3I clearance rales as il routine diagnostic test of Ihyroid dysfunction. J. Clin. Invest. 31, 141-58. 


\section{B. GEOFFROY et al.}

Blum M., Eisenbud M. (1967) Reduction of thyroid irradiation from 131-I by potassium iodide, JAMA 200. 1036-1040.

Castaing H., Fournet J.-P., Léger F.-A., Kiesgen F., Piette C., Dupard M.-C., Savoie J.-C. (1979) Thyrö̈de du nouveau-né et surcharge en iode après la naissance, Arch. Franç. Pédiat. 36, 356-368.

Cavalieri R.R. (1997) Iodine metabolism and thyroid physiology: current concepts, Thyroid 7, 177-81.

Clemens P.C., Neumann R.S. (1989) The Wolff-Chaikoff effect: hypothyroidism due to iodine application (letter), Arch. Dermatol. 125, 705.

Crocker D.G. (1984) Nuclear reactor accidents - the use of KI as a blocking agent against radioiodine uptake in the thyroid - a review, Health. Phys. 46, 1265-1279.

Cronquist A., Pochin E.E., Thompson B.D. (1971) The speed of suppression by iodate of thyroid iodine uptake, Health. Phys. 21, 393-394.

Cuddihy R.G. (1966) Thyroidal iodine-131 uptake, turnover and blocking in adults and adolescents, Health. Phys. 12, $1021-5$.

Delange F. (1990a) Iodine nutrition and risk of thyroid jrradiation from nuclear accidents. In: Iodine prophylaxis following nuclear accidents, (E. Rubery and E. Smales, Eds.) Proceedings of a joint WHO/CEC Workshop July 7988. pp. 45-56, Pergamon Press, Oxford.

Delange F. (1990b) The Wolff-Chaikoff Effect in Pediatrics. In: Iodine prophylaxis following nuclear accidents, (E. Rubery and E. Smales, Eds.) Proceedings of a joint WHO/CEC Workshop July 1988. pp. 83-92, Pergamon Press, Oxford.

Delange F. (1991) Blocage de l'activité thyroïdienne par l'iode stable. Cas particuliers de l'enfant et du foetus. In: Irradiation par l'iode radioactif. Joumée organisée par le comité de radiopro. tection d'Electricité de France, Paris, le 25 Octobre 1991, pp. 15-18. Jouve, Paris.

Delange F., Benker G., Caron P., Eber O., Ott W., Peter F., Podoba J., Simescu M., Szybinsky Z., Vertongen F., Vitti P., Wiersinga W., Zamrazil V. (1997) Thyroid volume and urinary iodine in European schoolchildren: standardization of values for assessment of iodine deficiency, Eur. J. Endocrinol. 136, $180-187$.

Evans T.C., Kretzschmar R.M., Hodges R.E., Song C.W. (1967) Radioiodine uptake studies of the human fetal thyroid, J. Nucl. Med. 8, 157-165.

Gaffney G.W., Gregerman R.I., Shock N.W. (1962) Relationship of age to the thyroidal accumulation, renal excretion and distribution of radioiodide in euthyroid man, JCEM 22, 784-794.

Hodges R.E., Evans T.C., Bradbury J.T., Keettel W.C. The accumulation of radioiodine by human fetal thyroids, J. Clin. Endocrinol. Metab. 15, 661-667.

International Agency for Atomic Energy. (1994) Intervention criteria in a nuclear or radiation emergency. Vienne, IAEA, Safety Series 109.

International Agency for Atomic Energy. (1996) International Basic Safety Standards for protection against ionizing radiation and for the safety of Radiation Sources. Vienne, IAEA, Safety Series 115.

ICRP Publication 30 (1979) Limits for intakes of radionuclides by workers, Ann. ICRP 2 (3-4).

ICRP Publication 56 (1990) Age-dependant doses to members of the public from intake of radionuclides: part 1, Ann. ICRP 20 (2).

ICRP Publication 63 (1991) Principles for intervention for protection of the public in a radiological emergency, Ann. ICRP 22 (4).

ICRP Publication 71 (1995) Age-dependent doses to members of the public from intake of radionuclides: part 4. Inhalation dose coefficients, Ann. ICRP 25 (3-4).

Johnson A.E. (1963) The rate of return of radioiodine uptake by the normal thyroid after suppression by pharmacological doses of stable iodide, Health Physics 9, 537-538.

Kaul A., Henrichs K., Roedler H.D. (1980) Radionuclide biokinetics and internal dosimetry in nuclear medicine, Ric. Clin. Lab. 10, 629-660.

Keating F.R., Albert A. (1949) The metabolism of iodine in man as disclosed with the use of radioiodine. In: Recent Progress in Hormone Research (G. Pincus, Ed.), pp. 429-481. Academic Press, New York.

Koutras D., Livadas D. (1966) The minimum dose of potassium iodide which inhibits the thyroidal radioiodine uptake, Nucl. Med. 5, 256-61. 
Koutras D.A., Alexander W.D., Buchanan W.W., Crooks J., Wayne E.J. (1960) Stable iodine metabolism in non toxic goitre, Lancet, 784-786.

Kovari M. (1994) Effect of delay time on effectiveness of stable iodine prophylaxis after intake of radioiodine, J. Radiol. Prot. 14, 131-136.

Lengemann F.W., Thompson J.C. (1963) Prophylactic and therapeutic measures for radioiodine contamination - a review, Health Physics 1391-1397.

Malarbet J.L., Aurengo A., Roy M., Le Guen B., Devillers C., Métivier H. (1998) Coefficients de dose après incorporation d'iode 129. Influence de l'apport alimentaire, Radioprotection 33, 15-33.

Malvaux P. Thyroid function during the neonatal period, infancy and childhood. (1985) In: Pediatric thyroidology (F. Delange et al., Eds.) pp. 33-43, Basel \& Karger.

Miller K.L., Coen P.E., White W.J., Hurst W.J., Achey B.E., Lang C.M. (1989) Effectiveness of skin absorption of tincture of $\mathrm{I}$ in blocking radioiodine from the human thyroid gland, Health Phys. 56, $911-914$.

Mochizuki Y., Mowafy R., Pasternack B. (1963) Weights of human thyroids in New York city, Health Phys. 9, 1299-1301.

Morgan A., Morgan D.J., Black A. (1968) A study of the deposition, translocation and excretion of radioiodine inhaled as iodine vapour, Health Phys. 15, 313-22.

Morgan D.J., Morgan A. (1967) Studies on the retention and metabolism of inhaled methyl iodide. I. Retention of inhaled methyl iodide, Health Phys. 13, 1055-1065.

Mornex R. (1987) Enquête sur la prévalence du goitre en France, Bull. Acad. Natl. Méd. 171, 301-306.

Myant N.B., Pochin E.E. (1949) The plasma iodide clearance rate of the human thyroid, Clin. Sc. 8, $109-131$.

Nauman J., Wolff J. (1993) Iodide prophylaxis in Poland after the Chernobyl reactor accident: benefits and risks, Am. J. Med. 94, 524-532.

National Council on Radiation Protection and Measurements, NCRP Publication 55 (1997) Protection of the thyroid gland in the event of releases of radioiodine. Washington: National Council on Radiation Protection and Measurements.

Noteboom J.L., Hummel W.A., Broerse J.J., de Vijlder J.J., Vulsma T., Jansen J.T., van Bekkum D.W. (1997) Protection of the matcrnal and fetal thyroid from radioactive contamination by the administration of stable iodide during pregnancy. An experimental evaluation in chimpanzees, Radiat. Res. 147, 691-697.

Noteboom J.L., Hummel W.A., Broerse J.J., de Vijlder J.J., Vulsma T, van Bekkum D. W. (1997) Protection of the infant thyroid from radioactive contamination by the administration of stable iodide. An experimental evaluation in chimpanzees, Radiat. Res. 147, 698-706.

Orgiazzi J. (1996) L'iode stable et la prévention des expositions à l'iode radioactif, Energies-Santé 7 , 379-385.

Oliner L., Kohlenbrener R.M., Fields T., Kunstadter R.H. (1957) Thyroid function studies in children: normal values for thyroidal 1131 uptake and PBIJ31 levels up to the age of 18, JCEM 17, 61-75.

Pahuja D.N., Rajan M.G., Borkar A.V., Samuel A.M. ( 1993) Potassium iodate and its comparison to potassium iodide as a blocker of 1311 uptake by the thyroid in rats, Health. Phys. 65, 545-549.

Parker R.H., Beierwaltes W.H. (1962) Elevated serum protein-bound iodine values with dietary iodine deficiency, JCEM 22, 19-25.

Piechowski J. (1997) Protection de la thyroïde en cas d'accident nucléaire ou rejet d'iode radioactif, Synaptique $\mathbf{5}$.

Pochin E.E., Barnaby C.F. (1962) The effect of pharmacological doses of non-radioactive iodide on the course of radioiodine uptake by the thyroid, Health Phys. 7, 125-126.

Pupi A., Becciolini A., Signorini C. (1991) Preventive Measures. Improvement of Practical Countermeasures Post-Chernobyl Actions. Luxembourg: CECEUR 12556.

Quimby E.H., Werner S.C.. Schmidt C. (1950) Influence of age, sex, and season upon radio-iodine uptake by the human thyroid, Proc. Soc. Exp. Biol. Med. 75, 537-540.

Ramsden D., Passant F.H., Peabody C.O., Speight R.G. (1967) Radioiodine uptakes in the thyroid. Studies of the blocking and subsequent recovery of the gland following the administration of stable iodine, Health Phys. 13, 633-646. 
Riggs D.S. (1952) Quantitative aspects of iodine metabolism in man, Pharmacol. Rev. 4, 284-370.

Ron E., Lubin J.H., Shore R.E., Mabuchi K., Modan B., Pottern L.M., Schneider A.B., Tucker M.A., Boice J.D., Jr. (1995) Thyroid cancer after exposure to external radiation: a pooled analysis of seven studies, Radiat. Res. 141, 259-277.

Rosenberg G. (1957) Effect of age on 24 hour iodine 131 uptake in healthy males, Canadian Services Medical Journal 13, 565-569.

Roti E., Gnudi A., Braverman L.E. (1983) The placental transport, synthesis and metabolism of hormones and drugs which affect thyroid function, Endocr. Rev. 4, 131-149.

Saxena K.M., Chapman E.M., Pryles C.V. (1962) Minimal dosage of iodide required to suppress uptake of iodine-131 by normal thyroid, Science 138, 430-431.

Schober B., Hunt J.A. (1976) Evaluation of the normal range of values for uptake of radioactive iodine by the thyroid gland, Can. Med. Assoc. J. 115, 29-35.

Shore R.E. (1992) Issues and epidemiological evidence regarding radiation-induced thyroid cancer, $R$ adiat. Res. 131, 98-111.

Stanbury J.B. (1990) The physiological basis for blockade of radioiodine retention by iodine. In: Iodine prophylaxis following nuclear accidents (E. Rubery and E. Smales, Eds.) pp. 57-64 Proceedings of a joint WHO/CEC Workshop July 1988. Pergamon Press, Oxford.

Sternthal E., Lipworth L., Stanley B., Abreau C., Fang S.L., Braverman L.E. (1980) Suppression of thyroid radioiodine uptake by various doses of stable iodide, N. Engl. J. Med. 303, 1083-1088.

Tubiana M., Bonniot R., Gelle X., Dutreix J., Pierquin B. (1958) Résultats de 3000 tests d'exploration fonctionnelle de la thyrö̈de par l'iode radioactif, La Presse Médicale 66, 64-47.

Tubiana M., Schlumberger M. (1997) In: Toxiques nucléaires (P. Gale and R. Paulin, Eds.) pp. 59-82. Masson, Paris.

Valeix P., Hercberg S. (1992) La déficience en iode: un problème de santé publique en France et dans les pays européens?, Cah. Nutr. Diét. 27, 24-31.

van Middlesworth L. (1963) Factors influencing the thyroid uptake of iodine isotopes from nuclear fission-a review, Health Phys. 9, 1197-1211.

Wang C., Crapo L.M. (1997) The epidemiology of thyroid disease and implications for sereening, Endocrinol. Metab. Clin. North. Am. 26, 189-218.

Weaver J.C., Kamm M.L., Dobson R.L. (1960) Excretion of radioiodine in human milk, JAMA 173, 872-875.

WHO/CEC. (1990) Report on the joint WHO/CEC Workshop on iodine prophylaxis following nuclear accidents. In: Iodine prophylaxis following nuclear accidents (E. Rubery and E. Smales, Eds.) pp. 151-169 Proceedings of a joint WHO/CEC Workshop July 1988. Pergamon Press, Oxford.

Wolff J. (1969) Iodide goiter and the pharmacologic effects of excess iodide, Am. J. Med. 47, 101-124.

Wootton R., Hammond B.J. (1978) A computer simulation study of optimal thyroid radiation protection during investigations involving the administration of radioiodine- labelled pharmaceuticals, Br. J. Radiol. 51, 265-272.

Zanconino P.B., Becker D.V. (1993) Use of potassium iodide to minimize thyroid radiation from radio active fall-out. In: Iodine deficiency in Europe, (F. Delange et al. Eds.). Plenum Press, New york.

Zvonova I.A. (1989) Dietary intake of stable I and some aspects of radioiodine dosimetry, Health Phys. 57, $471-475$. 